\title{
ASPECTOS RESPIRATORIOS, POSTURAIS E VOCAIS DA DOENÇA DE PARKINSON: CONSIDERAÇÕES TEÓRICAS
}

\section{Respiratory, Posture and Vocals Features in Parkinson's Disease - Theoretical Considerations}

\author{
Fernanda Vargas Ferreira ${ }^{(1)}$, Carla Aparecida Cielo ${ }^{(2)}$, Maria Elaine Trevisan ${ }^{(3)}$
}

\section{RESUMO}

Tema: manifestações respiratórias, posturais e vocais, associadas aos estágios da Doença de Parkinson (DP) e suas possíveis inter-relações. Objetivo: revisar a literatura, a fim de descrever as características respiratórias, posturais e vocais associadas aos estágios na DP e suas possíveis inter-relações. Conclusão: na DP, a etiologia das manifestações respiratórias é multifatorial, sendo obstrutivas; restritivas; relacionadas à bradicinesia, à rigidez e às alterações posturais; fraqueza da musculatura; anormalidades na musculatura laríngea; interferência da medicação antiparkisoniana. A postura em flexão altera a dinâmica corporal, interferindo nas funções estomatognáticas e na relação tóraco-abdominal. As alterações vocais podem estar presentes desde os estágios iniciais com diminuição dos tempos máximos de fonação e da intensidade vocal; adução glótica incompleta; fendas glóticas; tremor e instabilidade fonatória; perturbações de frequência e de intensidade; e qualidade vocal alterada. Os déficits na força muscular respiratória se refletem no menor suporte respiratório à emissão vocal e, por consequência, na diminuição da intensidade vocal e dos tempos máximos de fonação. Do mesmo modo, a postura em flexão gera desvantagem biomecânica à atividade respiratória, com menor expansão e redução dos volumes pulmonares, e déficit na excursão diafragmática. Os distúrbios respiratórios, posturais e/ou vocais, à medida que a DP evolui, de acordo com os seus estágios, tendem ao maior comprometimento, apresentando efeitos na comunicação verbal e não-verbal.

DESCRITORES: Doença de Parkinson; Voz; Respiração; Postura

\section{INTRODUÇÃO}

A Doença de Parkinson (DP) é uma enfermidade neurológica progressiva e idiopática ${ }^{1,2}$ cujas

(1) Fisioterapeuta; Professora do Curso de Fisioterapia do Centro Universitário Franciscano, UNIFRA, Santa Maria, RS; Mestre em Distúrbios da Comunicação Humana pela Universidade Federal de Santa Maria.

(2) Fonoaudióloga; Professora Adjunta do Departamento de Fonoaudiologia da Universidade Federal de Santa Maria, UFSM, Santa Maria, RS; Doutora em Lingüística Aplicada pela Pontifícia Católica do Rio Grande do Sul.

(3) Fisioterapeuta; Professora Assistente do Departamento de Fisioterapia e Reabilitação da Universidade Federal de Santa Maria, UFSM, Santa Maria, RS; Mestre em Ciência do Movimento Humano - Área de Fisiologia do Exercício pela Universidade Federal de Santa Maria.

Programa de Pós-Graduação em Distúrbios da Comunicação Humana. Universidade Federal de Santa Maria (UFSM). Santa Maria, RS, Brasil

Conflito de interesses: inexistente características são tremor em repouso, bradicinesia, rigidez, alterações posturais e de equilíbrio, depressão e disartrofonia hipocinética ${ }^{1,3,4}$. Ela afeta homens e mulheres, na maioria das vezes após os 50 anos de idade ${ }^{2,4}$.

Suas repercussões ocorrem em todos os sistemas, incluindo o respiratório, o músculoesquelético e o estomatognático. As disfunções respiratórias, de etiologia multifatorial, ocorrem na maioria dos pacientes com DP em estágios avançados ${ }^{5,6}$. A força muscular respiratória apresenta grau de comprometimento compatível com o estágio da DP, evidenciando que as condições funcionais respiratórias decrescem proporcionalmente à evolução da doença $5,7,8$.

Em relação à postura corporal, o parkinsoniano típico apresenta "postura em flexão", caracterizada por flexão da cabeça, hipercifose dorsal, protração e abdução de ombros e flexão dos braços ${ }^{1,4,8,9}$. Autores comentam que os distúrbios posturais de 
cabeça alteram a movimentação da articulação temporomandibular, elemento importante para a fonação ${ }^{1,9}$.

A disartrofonia hipocinética acomete em torno de $75 \%$ dos parkinsonianos e caracteriza-se por monotonia de frequência e de intensidade, articulação imprecisa, disprosódia e alterações na velocidade, o que tende a repercutir na expressão oral do indivíduo ${ }^{1,3,9}$.

Com base nessas premissas, o objetivo do presente trabalho foi revisar a literatura, a fim de descrever as características respiratórias, posturais e vocais associadas aos estágios na DP e suas possíveis inter-relações.

\section{MÉTODO}

Para esta revisão de literatura, realizou-se um levantamento bibliográfico compreendendo o período de 1984 até 2009, utilizando livros, monografias, artigos de periódicos e Internet. Nesta última, foram pesquisados artigos pelas bases de dados Lilacs, BIREME, PubMed, MedLine, Scielo e Google Schoolar. A consulta foi sistemática, em fontes clássicas e atuais da literatura científica nacional e internacional.

Para a busca, utilizaram-se os termos "Doença de Parkinson", "respiração", "postura corporal", "voz" e "estágios da Doença de Parkinson". Foram incluídos os estudos relevantes para o objetivo da pesquisa.

Os critérios de inclusão adotados para a seleção dos materiais bibliográficos foram: publicações científicas; apresentar abordagens a respeito da DP e ou de suas características e estágios; apresentar abordagens sobre respiração, postura corporal e voz na DP, correlacionadas ou não; apresentar data de publicação dentro do período de 1984 a 2009.

A partir da pesquisa realizada com os unitermos e com os critérios citados, foram selecionadas 50 referências bibliográficas, sendo que, dessas, são 07 livros, 26 artigos internacionais e 17 artigos nacionais.

Os resultados da busca foram organizados, apresentados e discutidos em diferentes itens, priorizando a elucidação do objetivo proposto e a apresentação do assunto aos profissionais fisioterapeutas e fonoaudiólogos.

\section{REVISÃO DA LITERATURA}

\section{Disfunções respiratórias na Doença de Parkinson}

Disfunções do sistema respiratório têm sido identificadas como manifestações comuns nos estágios mais avançados da $\mathrm{DP}^{5,10}$, no entanto, a força muscular respiratória e seus efeitos nas atividades de vida diária e na qualidade de vida dos indivíduos não têm sido muito enfatizados nas pesquisas.

A etiologia das disfunções respiratórias permanece desconhecida, sendo considerada multifatorial, possivelmente em virtude da fisiopatologia complexa, dos poucos estudos investigativos e, ainda, do fato de que as suas repercussões podem ser assintomáticas. Entretanto, alguns autores sugerem hipóteses acerca da disfunção respiratória na DP e suas consequências no dia-a-dia do parkinsoniano, ilustradas por: a) distúrbios no padrão ventilatório e respiratório, b) déficit na força dos músculos respiratórios com fraqueza muscular respiratória, c) obstrução crônica das vias aéreas ${ }^{6,11}$, d) ação simultânea de fatores como o grau de bradicinesia ou rigidez e limitações músculo-esqueléticas da coluna vertebral $5,7,11$, e) anormalidades na musculatura laríngea ${ }^{1,12}$; f) discinesias musculares produzidas pela levodopa $4,6,8$.

Um aspecto relevante da disfunção respiratória na DP é a força muscular respiratória. Em algumas concepções ${ }^{7,8,13}$, a fraqueza da musculatura respiratória, especialmente da musculatura expiratória, pode ocasionar redução da pressão expiratória máxima, menores fluxos expiratórios, bem como aumento do volume residual, possivelmente correlacionados ao agravamento da doença.

Estudo brasileiro ${ }^{11}$ comparou o padrão respiratório de dez indivíduos com DP e de dez idosos assintomáticos, entre 60 e 75 anos de idade. Cada um dos grupos foi composto por cinco homens e cinco mulheres, submetidos à pletismografia respiratória por indutância. Como resultados, os parkinsonianos apresentaram redução do volume corrente e do fluxo inspiratório médio e frequência respiratória maior, resultando numa ventilação/ minuto significativamente menor, quando comparados aos idosos assintomáticos.

A partir do exposto, é possível que a diminuição da força dos músculos respiratórios tenda a restringir as atividades de vida diária (AVD's), bem como a prática de exercícios físicos e a fonação.

\section{Alterações Posturais na Doença de Parkinson}

A instabilidade postural é considerada, atualmente, um componente da chamada tétrade característica da DP, uma vez que as alterações posturais encontram-se presentes desde o início da patologia, tendendo ao agravamento conforme a evolução da doença ${ }^{4,11,14}$.

A postura clássica do parkinsoniano é a de flexão da cabeça, hipercifose torácica, protração e abdução de ombros e flexão dos braços. Os reflexos posturais são inadequados, e o equilíbrio pode ser perdido facilmente ${ }^{4,15}$. 
Em estudo com cinco indivíduos com DP, sendo quatro homens e uma mulher, entre 37 e 53 anos de idade, observou-se, por meio de avaliação postural subjetiva, que todos apresentaram hiperlordose cervical com flexão da cabeça, que quatro dos cinco sujeitos apresentaram hipercifose dorsal e que quatro sujeitos apresentaram protrusão de ombros ${ }^{16}$.

Alterações posturais de cabeça limitam os movimentos da mandíbula e da própria cabeça, interferindo na produção vocal ${ }^{17}$. Segundo a literatura, a posição anteriorizada de cabeça, presente na DP e no respirador oral $(\mathrm{RO})$, por exemplo, altera as relações biomecânicas, crâniocervicais e craniomandibulares e, consequentemente, as funções estomatognáticas, especialmente a fonação ${ }^{1,17-19}$.

O aumento do esforço inspiratório, em função da alteração postural, desencadeia o padrão ventilatório apical, elevando os potenciais de ação de músculos como o esternocleidomastóideo, resultando em encurtamento, perda de flexibilidade e alterações no posicionamento da cabeça ${ }^{20,21}$. O mesmo ocorre com o diafragma, que se fixa às costelas e às vértebras lombares, recebendo apoio dos músculos abdominais na zona de aposição, favorecendo sua excursão. Por consequência, qualquer alteração postural pode repercutir na dinâmica tóraco-abdominal ${ }^{18,22}$, na respiração e na fonação.

\section{Aspectos Vocais na Doença de Parkinson}

$\mathrm{Na}$ DP, são comumente afetadas as funções fonatória, articulatória e respiratória, devido à rigidez e à bradicinesia, resultando em desordens da fala ${ }^{23,24}$, devido à fraqueza, à alteração da mobilidade, à atrofia e à fadiga muscular presentes na doença ${ }^{9,25}$. Os distúrbios da voz e da fala na DP variam de caso para caso, sendo que suas características decorrem da redução dos movimentos dos órgãos fonoarticulatórios, incluindo a laringe, repercutindo na ausência de sincronia entre os movimentos e o controle temporal ${ }^{26}$.

Em relação ao prejuízo peculiar da expressão verbal do parkinsoniano, as alterações fonoaudiológicas tendem a aumentar sua gravidade e frequência de ocorrência com a duração e evolução da doença ${ }^{27,28}$, sendo que tais desordens podem estar presentes em fases precoces da enfermidade, inclusive como o primeiro indício do dano neurológico ${ }^{1,26,29}$.

Entre as manifestações da disartrofonia hipocinética, destacam-se os decréscimos dos tempos máximos de fonação (TMF) e da intensidade vocal ${ }^{29-31}$, que tendem a tornar a expressão oral do parkinsoniano ininteligível. Sabe-se que a medição dos TMF fornece medidas pneumofônicas e de qualidade de voz, relacionando a habilidade do indivíduo em controlar as forças aerodinâmicas da corrente aérea pulmonar e as forças mioelásticas da laringe ${ }^{32,33}$. A intensidade vocal depende basicamente do aumento da pressão de ar subglótica, controlada pela adução glótica e pelo fluxo aéreo expiratório ${ }^{32,34}$. Os déficits de TMF e de intensidade vocal decorrem possivelmente da rigidez muscular e da bradicinesia, que se refletem por meio da adução glótica incompleta, descontrole do movimento laríngeo, tremor vocal, dificuldades de sonorização e respiratórias ${ }^{29,30,35,36}$.

Com base nessas considerações, verifica-se que a integridade estrutural e a harmonia funcional entre os músculos laríngeos e respiratórios são de vital importância para a correta e inteligível produção vocal. Ressalta-se a magnitude do controle neural da voz, imprescindível para que haja a integração entre os sistemas nervoso central, periférico e límbico, comprometido na DP.

Entre toda a gama de sinais e sintomas presentes na DP, distúrbios vocais têm sido considerados impactantes na vida do parkinsoniano, no entanto, a etiologia permanece desconhecida, havendo extensa variabilidade das características vocais acústicas, aparentemente com pouca correspondência com a severidade da doença ${ }^{1,26,37}$.

Estudos referem que algumas características da DP podem justificar a variedade de achados acústicos da voz desses pacientes como o fechamento glótico incompleto com arqueamento das pregas vocais que pode gerar fendas glóticas triangulares ou do tipo fusiforme ${ }^{1,35-38}$; a presença de tremor ${ }^{30,36}$; a rigidez da musculatura laríngea ${ }^{2,34,38}$; e a instabilidade vibratória das pregas vocais ${ }^{32,35,36}$.

Como principais achados nos estudos da voz dos parkinsonianos, encontraram-se: alteração da f0, por meio do GLIMPES em oito parkinsonianos, no período ON, obtendo-se a f0 de $128 \mathrm{~Hz}{ }^{39}$; f0 média baixa, de $128 \mathrm{~Hz}$ em oito homens com DP ${ }^{40}$; média de fo de $150,8 \mathrm{~Hz}$ para os homens em 24 indivíduos com DP, através do Computerized Speech Lab, nas fases ON e OFF ${ }^{35}$; média de f0 de $194 \mathrm{~Hz}$, através do Computerized Speech Lab, em vinte parkinsonianos na fase $\mathrm{ON}^{41}$; alteração nos parâmetros de perturbação da frequência e da intensidade $^{29,35,39}$; e qualidade vocal alterada, especificamente rouquidão, aspereza e soprosidade ${ }^{29,30,32,35,36}$.

\section{Inter-relações entre achados respiratórios, posturais e vocais na Doença de Parkinson, conforme seus estágios}

A voz é uma função adaptada do ser humano, cujo resultado é a linguagem oral, utilizada para, entre outros fins, promover socialização. Sua complexidade advém da interação anatomofisiológica entre as estruturas centrais, que vão gerar o ato 
motor da fonação; as estruturas moduladoras, que são o sistema límbico, gânglios da base e tálamo; e, por fim, os órgãos efetores, músculos que executarão a tarefa a partir dos estímulos nervosos periféricos ${ }^{30,32,34}$.

Estudos apontam que déficits na produção vocal podem se originar da perda da capacidade respiratória, achado comum na DP, uma vez que essa função é fonte de energia para a vocalização. A rigidez característica da DP propicia o encurtamento dos sarcômeros musculares, comprometendo a atividade contrátil nas fases inspiratória e expiratória, gerando deterioração da força muscular respiratória ${ }^{4,11}$. Deste modo, os músculos respiratórios atuam de forma limitada, bem como os músculos laríngeos adutores e tensores ${ }^{32,34}$, gerando alterações dos TMF e da qualidade vocal como verificado na literatura ${ }^{29,30,35}$.

Em pesquisa, avaliaram-se vinte e quatro parkinsonianos, encontrando-se média de TMF de 11,64s para os homens e 12,27s para as mulheres ${ }^{35}$. Em estudo com vinte e oito parkinsonianos, com idades entre 61 e 78 anos, encontrou-se, como médias de TMF para os homens e mulheres com DP, respectivamente, 10 e 7 segundos(s) ${ }^{29}$. Investigaram-se cinco pacientes com DP, entre 69 e 90 anos, cujos resultados apontaram decréscimo nos TMF de /a/; /s/; /z/ e na relação s/z ${ }^{30}$.

Alterações da intensidade vocal, consideradas um dos sintomas mais impactantes na vida do parkinsoniano, apresentaram-se em diversos estudos, tendendo ao decréscimo e à redução da inteligibilidade 1,29,30,35,37.

Tais resultados também são convergentes com estudo realizado com vinte e oito parkinsonianos, na fase pré-tratamento, cuja intensidade vocal mostrou-se reduzida no nível de $65 \mathrm{~dB}^{29}$ e com os resultados de pesquisa com cinco indivíduos com $\mathrm{DP}$, em que a intensidade média foi de $60 \mathrm{~dB}^{30}$, bem como em estudo com dois parkinsonianos, cuja intensidade média foi de $60,5 \mathrm{~dB}^{42}$.

Além da diminuição da força muscular respiratória e do fluxo expiratório máximo atuarem como fatores interferentes na emissão vocal, a ausência ou diminuição do controle respiratório na DP, especialmente a incoordenação tóraco-abdominal, reflete-se nos distúrbios da voz, o que tende a inviabilizar a comunicação $0^{11,43}$.

Os aspectos da força de contração da musculatura esquelética são determinados pelo comprimento do músculo pré-contração, encurtamento, força e freqüência da estimulação e integridade do mecanismo de contração ${ }^{44,45}$, encontrando-se comprometidos na DP, uma vez que a imobilidade e a rigidez generalizada dos músculos responsáveis pela respiração dificultam a expansão do conjunto tórax-abdômen, bem como diminuem a força muscular ${ }^{11,12}$, podendo alterar a produção vocal.

A força muscular respiratória está relacionada aos volumes pulmonares, especialmente a força do diafragma, principal músculo inspiratório. Alguns autores referem que o encurtamento do diafragma e dos intercostais ocasiona mudanças de volume pulmonar ${ }^{1,46}$, influenciando os TMF e a qualidade da voz, principalmente a intensidade.

Outro ponto a se considerar é a influência da postura corporal na biomecânica respiratória, já que a postura em flexão do parkinsoniano gera restrição à excursão diafragmática, especialmente pela hiperlordose lombar ${ }^{4,11,16}$. Ainda, a rigidez muscular e a bradicinesia dificultam a capacidade de distensibilidade dos pulmões e de variações de pressão e de volume aéreos, afetando a produção da voz e da fala ${ }^{1,8}$.

Em virtude dos desvios posturais típicos da DP, é possível que o parkinsoniano apresente maior gasto de energia e maior esforço, uma vez que a posição da mandíbula e do crânio, da coluna cervical, das estruturas supra e infrahioídeas, da cintura escapular e da coluna torácica e lombar atuam como uma unidade biomecânica, de modo que alterações em alguns desses componentes podem desencadear alterações nos sistemas músculo-esquelético e estomatognático ${ }^{16,47,48}$. Ainda, a hiperatividade muscular ocasiona um deslocamento para frente da região cérvico-escapular, interferindo na fonação ${ }^{49,50}$.

Ainda em relação às alterações posturais no quadrante superior, a hipercifose torácica restringe a expansão do gradil costal e a hiperlordose lombar, associada geralmente à protrusão abdominal, prejudica as capacidades respiratórias e a dinâmica diafragmática necessárias à emissão vocal, especialmente em circunstâncias que requeiram maior projeção vocal ${ }^{4,11,16}$.

Assim, a partir das complexas conexões neurais, estruturais e funcionais que inter-relacionam os sistemas respiratório, músculoesquelético e estomatognático, constituindo a unidade corpovoz-respiração, qualquer desarmonia repercute negativamente, resultando em menor habilidade de comunicação verbal e não-verbal no indivíduo com DP.

Ainda, sugere-se novas pesquisas em virtude da complexidade dos sinais e sintomas da doença, bem como da escassez de estudos interdisciplinares sobre o tema.

\section{CONCLUSÃO}

$\mathrm{Na}$ DP, a maioria dos autores concorda que a etiologia das manifestações respiratórias é 
multifatorial, sendo as possíveis causas de ordem obstrutiva; restritivas; relacionadas à bradicinesia, à rigidez e às alterações posturais; fraqueza da musculatura respiratória; anormalidades na musculatura laríngea e interferência da medicação antiparkisoniana. Ainda, o déficit da força muscular respiratória, ilustrado por menores fluxos expiratórios, é uma das principais limitações funcionais decorrentes dos distúrbios respiratórios nesta doença.

A literatura refere que a postura em flexão da DP altera a dinâmica corporal como um todo, interferindo nas funções estomatognáticas, em virtude especialmente da anteriorização da cabeça; e interfere na relação tóraco-abdominal, com a hipercifose dorsal e a hiperlordose lombar refletindo-se negativamente na expansão da caixa torácica e na excursão diafragmática, respectivamente.

$\mathrm{Na} \mathrm{DP}$, conforme a literatura pesquisada, as alterações vocais podem estar presentes desde os estágios iniciais com diminuição dos tempos máximos de fonação e da intensidade vocal, possivelmente pela rigidez e bradicinesia; adução glótica incompleta; fendas glóticas; tremor e instabilidade fonatória; perturbações de frequência e de intensidade; e qualidade vocal alterada.

A partir desta revisão de literatura, pode-se considerar que os déficits na força muscular respiratória da DP se refletem no menor suporte respiratório à emissão vocal e, por consequência, na diminuição da intensidade vocal e dos tempos máximos de fonação. Do mesmo modo, a postura em flexão gera desvantagem biomecânica à atividade respiratória, com menor expansão e redução dos volumes pulmonares, e déficit na excursão diafragmática.

Pôde-se verificar ainda que os distúrbios respiratórios, posturais e/ou vocais, à medida que a DP evolui, de acordo com os seus estágios, tendem ao maior comprometimento, apresentando efeitos na comunicação verbal e não-verbal.

\begin{abstract}
Background: respiratory, postural and vocal manifestations, associated to stages in Parkinson's Disease and their possible interrelations. Purpose: to review the literature in order to describe breathing, posture and voice characteristics associated with stages in PD and their possible interrelations. Conclusion: etiology of respiratory disease is multifactorial in PD, such as obstructive, restrictive, related to bradykinesia, rigidity and postural changes, muscle weakness, abnormalities of the laryngeal muscles; antiparkisonian impact from medication. Flexion position alters body dynamics, interfering in the stomatognathic functions and thoracoabdominal relationships. Vocal changes may come occur from the early stages with a decrease in maximum phonation times and vocal intensity, incomplete glottic adduction, glottic chink, vocal tremor and instability; disturbances of frequency and intensity, and changes in voice quality. Deficits in respiratory muscle strength are reflected in the lower respiratory support to vocal production and consequently in decrease of vocal intensity and maximum phonation times. Similarly, the flexion position generates biomechanical disadvantage concerning respiratory activity, with lower growth and reduced lung volumes, and deficits in diaphragmatic excursion. Respiratory, postural and / or vocal disorders, as PD progresses, according to their stages, tend to show greater impairment, demonstrating effects on verbal and nonverbal communication.
\end{abstract}

KEYWORDS: Parkinson Disease; Voice; Respiration; Posture

\section{REFERÊNCIAS}

1. Schulz GM, Grant MK. Effects of speech therapy and pharmacologic and surgical treatments on voice and speech in Parkinson's disease: a review of the literature. J Commun Disord. 2000; 33(1):59-88.

2. Silverman EP, Sapienza CM, Carmichael C, Davenport PW, Hoffman-Ruddy B, Okun MS. Tutorial on maximum inspiratory and expiratory mouth pressures in individuals with Idiopathic
Parkinson disease (IPD) and the preliminary results of an expiratory muscle strength training program. Neuro Rehabil. 2006; 21(1):71-9.

3. Lamônica DAC, Saes SO, Paro PMM, Brasolotto AG, Soares-Barbosa A. Doença de Parkinson: proposta de protocolo de anamnese. Salusvita. 2003; 22(3):363-71.

4. André ES. Moléstia de Parkinson. Fisiot em Mov. 2004; 17(1):11-24. 
5. Haas, BM, Trew M, Castle PC. Effects of respiratory muscle weakness on daily living function, quality of life, activity levels and exercise capacity in mild to moderate Parkinson's disease. Austr $\mathrm{J}$ Physical Med and Rehab. 2004; 83(8):601-7.

6. Alves LA, Coelho AC, Brunetto AF. Fisioterapia respiratória na doença de Parkinson idiopática: relato de caso. Fisiot Pesq. 2005; 12(3):46-9.

7. Bogaard JM, Hovestadt A, Meerwaldt JVD. Maximal expiratory and inspiratory flow-volume curves in Parkinson's disease. Am Rev Resp Disease. 1989; (139):610-4.

8. Cardoso SR, Pereira JS. Distúrbio respiratório na doença de Parkinson. Fisiot Brasil. 2000; 1(1):23-6.

9. Carro OT, León M, Alvarez E, Alvarez L, Maragato C, Rireva O. Particularidades articulares de la disartria Parkinsoniana. Rev Mex Neurociência. 2001; 2(4):235-9.

10. Shiermeier S, Schäfer D, Schäfer T, Greulich W, Schläfke ME. Breathing and locomotion in patients with Parkinson's disease. Eur J Physiology. 2001; 443(1):67-71.

11. Parreira VF, Guedes LU, Quintão DG, Silveira EP, Tomich GM, Sampaio RF, et al. Padrão respiratório em pacientes portadores da doença de Parkinson e em idosos assintomáticos. Acta Fisiátrica. 2003; 10(2):61-6.

12. Tamaki A, Matsuo $Y$, Yanagihara T, Abe K. Influence of thoracoabdominal movement on pulmonary function in patients with Parkinson's Disease: comparison with healthy subjects. Neurorehabil Neural Repair. 2000; 14(1):43-7.

13. Saleem AF, Sapienza CM, Okun MS. Respiratory muscle strength training: treatment and response duration in a patient with early idiophatic Parkinson's disease. Neuro Rehabil. 2005; 20(4):323-33.

14. Bartolic A, Pirtosek Z, Rozman J, Ribaric S. Postural stability of Parkinson's disease patients is improved by decreasing rigidity. Eur J Neurol. 2005; 12(2):156-9.

15. Fahn S. Description of Parkinson's disease as clinical syndrome. Ann N Y Acad Sci. 2003; (991):1-14.

16. Ferreira FV, Prado ALC, Cielo CA, Busanello AR. A relação da postura corporal com a prosódia na doença de Parkinson: estudo de caso. Rev. CEFAC. 2007; 9(3):319-29.

17. Ferreira LP, Befi-Lopes DM, Limongi SC, organizadores. Tratado de fonoaudiologia. São Paulo: Roca; 2004.

18. Carvalho GD. S.O.S. Respirador bucal. São Paulo: Lovise; 2003. 286p.

19. Tessitore A. Regulação orofacial: sua importância no equilíbrio das funções estomatognáticas. Anais do $16^{\circ}$ Conclave Internacional de Campinas; março/ abril 2005. p. 1-7.
20. Corrêa ECR, Bérzin F. Mouth breathing syndrome: cervical muscles recruitment during nasal inspiration before and after respiratory and postural exercises on Swiss Ball. Int J Pediatric Otorhinolaryngol. 2008; 72(9):1335-43.

21. Pasinato F, Rlbeiro-Corrêa E, Peroni ABF. Avaliação da mecânica ventilatória em indivíduos com disfunção têmporomandibulare assintomáticos. Rev Bras Fisio. 2006; 10(3):285-9.

22. Ribeiro-Corrêa E, Bérzin F. Temporomandibular disorder and dysfunctional breathing. Braz $\mathrm{J}$ Oral Sciences. 2004; 3(2):498-502.

23. Carrara-De-Angelis E, Mourão LF, Ferraz HB, Behlau MS, Pontes PA, Andrade LA. Effect of voice rehabilitation on oral communication of Parkinson's disease patients. Acta Neurol Scand. 1998; 96(4):199-05.

24. Jiang J, O'Mara T, Chen H, Stern JI, Vlagos D, Hanson D. Aerodynamic measurements of patients with Parkinson's disease. J Voice. 1999; 13(4):583-91 .

25. Gasparini G, Diaféria G, Behlau M. Queixa vocal e análise perceptivo-auditiva de pacientes com Doença de Parkinson. Rev Ciê Méd Biol. 2003; 2(1):72-6.

26. Locco J. La production des occlusives dans la maladie de Parkinson. [tese] França: L'Universite Aix-Marseille; 2005. 338p.

27. Deane KH, Whurr R, Playford ED, Ben-Shlomo $Y$, Clarke CE. Speech and languagen therapy for dysarthria in Parkinson's disease. Cochrane Database Syst Rev. 2001 [cited 2001 Apr]; (2): [about CD002812 - CK002814). Disponível em: http://mrw.interscience.wiley.com/cochrane/ clsysrev/articles/CD002814/frame.html.

28. Holmes RJ, Oates JM, Phyland DJ, Hughes AJ. Voice characteristics in the progression of Parkinson's Disease. Int J Lang Commun Disord. 2000; 35(3):407-18.

29. Dias AE, Limongi JCP. Tratamento dos distúrbios da voz na doença de Parkinson, o método Lee Silverman. Arq Neuro-Psiquiatr. 2003; 61(1):61-6.

30. Silveira DN, Brasolotto AG. Reabilitação vocal em pacientes com doença de Parkinson: fatores interferentes. Pró-Fono. 2005; 17(2):241-50.

31. Jöbges EM, Spittler-Schneiders H, Renner CIE, Hummelsheim $\mathrm{H}$. Clinical relevance of rehabilitation programs for patients with idiopathic Parkinson syndrome. II: Symptom-specific therapeutic approaches. Parkinsonism and related disorders. 2007; 13(4):203-13.

32. Pinho SMR. Fisiologia da fonação. In: Ferreira LP, Befi-Lopes DM, Limongi SCO. Tratado de fonoaudiologia. São Paulo: Roca; 2004. p. 3-10.

33. Rossi DC, Munhoz DF, Nogueira CR, Oliveira TCM, Britto ATB. Relação do pico de fluxo 
expiratório com o tempo de fonação em pacientes asmáticos. Rev. CEFAC. 2006; 8(4):509-17.

34. Behlau MS. A voz que ensina. Revinter: Rio de Janeiro; 2005. 68p.

35. Carrara-De-Angelis E. Deglutição, configuração laríngea, análise clínica e análise computadorizada da voz de pacientes com doença de Parkinson. [tese]. São Paulo (SP): Universidade Federal de São Paulo; 2000.

36. Cervantes $O$. Doenças neurológicas: repercussões laríngeas e vocais. In: Dedivitis RA, Barros APB. Métodos de avaliação e diagnóstico de laringe e voz. São Paulo: Lovise; 2002. 145-75.

37. Yucertuk AV, Yilmaz H, Egrilmez M, Karaca S. Voice analysis and videolaryngostroboscopy in patients with Parkinson's disease. Eur Arch Otorhinolaryngol. 2002; 259(6):290-3.

38. Gallena S, Smith PJ, Zeffiro T, Ludlow CL. Effects of levodopa on laryngeal muscle activity for voice onset and offset in Parkinson Disease. J Speech Lang Hear Res. 2001; 44(6):1284-99.

39. Titze IR. Parameterization of the glottal area, glottal flow and vocal fold contact area. J Acoust Soc Am. 1984; 75(2):570-80.

40. Ramig LO, Titze IR, Scherer RC, Ringel SP. Acoustic analysis of voices of patients with neurologic disease: rationale and preliminary data. Ann Otol Rhinol Laryngol. 1988; 97(2pt1):164-72.

41. Sanabria J, Ruiz PG, Gutierrez R, Marquez F, Escobar P, Gentil M, et al. The effect of Levodopa on vocal function in Parkinson's disease. Clin Neuropharmacol. 2001; 24(2):99-102.

42. Rosa JC, Cielo CA, Cechella C. Função fonatória em pacientes com Doença de Parkinson: uso de instrumento de sopro. Rev. CEFAC. 2009;11(2):305-13.

43. Vitorino MR, Homem FCB. Doença de Parkinson: da fonação à articulação. Fono Atual. 2001; 4(17):35-9.

44. Silva LC, Rubin AS, Silva LMC. Avaliação funcional pulmonar. Rio de Janeiro: Revinter; 2000. 45. Di Lorenzo VA, Silva AB, Sampaio LMM, Jamami M, Oishi J, Costa D. Efeitos do treinamento físico e muscular respiratório em pacientes com Doença Pulmonar Obstrutiva Crônica (DPOC) grave submetidos a BiPAP. Rev Bras Fisio. 2003; 7(1):69-76.

46. Gemelli M, Pegoraro M, Nakayama GK, Machado JL, Carvalho AR. Avaliação da influência de técnicas fisioterapêuticas manuais sobre as pressões inspiratória e expiratória em atletas de natação. Reabilitar. 2005; 7(28):35-41.

47. Tedeschi-Marzola F, Marques AP, Marzola C. Contribuição da Fisioterapia para a Odontologia nas disfunções da articulação temporomandibular. Rev Odonto Ciência. 2002; 17(36):119-34.

48. Arboleda BMW, Frederick AL. Considerations for maintenance of postural alignment for voice production. J Voice. 2008; 22(1):90-9.

49. Yi LC, Guedes ZCF, Vieira MM. Relação da postura corporal com a disfunção da articulação tempomandibular: hiperatividade dos músculos mastigatórios. Fisio Brasil. 2003; 4(5):341-7.

50. Amantéa DV, Novaes AP, Campolongo GD, Barros TP. A importância da avaliação postural no paciente com disfunção da articulação temporomandibular. Acta Ortopéd Bras. 2004; 12(3):155-9.

doi: $10.1590 / S 1516-18462010005000135$

RECEBIDO EM: 24/08/2009

ACEITO EM: 06/09/2010

Endereço para correspondência:

Fernanda Vargas Ferreira

Rua Dr. Maia 4059, Centro

Uruguaiana, RS

CEP: 97510-163

E-mail: nandaf_pg@yahoo.com.br 